\title{
Plasma thymidylate synthase and dihydrofolate reductase mRNA levels as potential predictive biomarkers of pemetrexed sensitivity in patients with advanced non-small cell lung cancer
}

\author{
Tao Li ${ }^{1 \#}$, Jin-Bo Huang ${ }^{2 \#}$, Jun-Guo Lu ${ }^{1}$, Rong $\mathrm{Li}^{3}$, Yan Wang ${ }^{1}$, Xiang-Rong Shi ${ }^{1}$, Min-Xin Shi ${ }^{4}$, \\ Xiao-Dong Zhang ${ }^{1}$
}

${ }^{1}$ Department of Medical Oncology, Nantong Tumor Hospital, Nantong, China; ${ }^{2}$ Department of Respiratory Medicine, Affiliated Hospital of Nantong University, Nantong, China; ${ }^{3}$ Department of Medical oncology, Affiliated Taikang Xianlin Drum Tower Hospital, Medical School of Nanjing University, Nanjing, China; ${ }^{4}$ Department of Thoracic Surgery, Nantong Tumor Hospital, Nantong, China

Contributions: (I) Conception and design: XD Zhang, MX Shi; (II) Administrative support: None; (III) Provision of study materials or patients: None; (IV) Collection and assembly of data: All authors; (V) Data analysis and interpretation: All authors; (VI) Manuscript writing: All authors; (VII) Final approval of manuscript: All authors.

"These authors contributed equally to this work.

Correspondence to: Min-Xin Shi. Department of Thoracic Surgery, Nantong Tumor Hospital, Nantong, China. Email: wangzhiqing@mail.sdu.edu; Xiao-Dong Zhang. Department of Medical Oncology, Nantong Tumor Hospital, Nantong, China. Email: cnbqem79@163.com.

Background: High levels of thymidylate synthase (TS) and dihydrofolate reductase (DHFR) expression in tumour tissues are an indicator of ineffective responses to pemetrexed-based chemotherapy in various tumours, including non-small cell lung cancer (NSCLC). However, tumour tissues are highly heterogeneous, so a single biopsy may not reflect genetic alterations during disease progression. This study investigated the potential use of plasma TS and DHFR mRNA levels as biomarkers for predicting sensitivity to pemetrexedbased chemotherapy.

Methods: Plasma samples were obtained from 245 patients with advanced NSCLC and 30 healthy donors. Total RNA was extracted from the plasma samples, and TS and DHFR mRNA levels were determined via real-time PCR. TS and DHFR mRNA levels between cancer patients and healthy controls were compared. The association between plasma TS and DHFR mRNA levels and tumour response to pemetrexed/cisplatin chemotherapy was analysed.

Results: The plasma TS and DHFR mRNA levels decreased in patients with advanced NSCLC compared with healthy controls. Moreover, plasma TS and DHFR mRNA levels negatively correlated with tumour response to pemetrexed/cisplatin chemotherapy in patients with advanced NSCLC. Overall survival time was prolonged in patients with low TS mRNA expression compared with those with high TS mRNA expression, although the difference was not statistically significant.

Conclusions: Low expression levels of plasma TS and DHFR mRNA confer increased tumour sensitivity to pemetrexed/cisplatin chemotherapy in patients with advanced NSCLC. The results suggested that plasma TS and DHFR mRNA levels are promising biomarkers for choosing patients who are likely to respond and benefit the most from pemetrexed-based chemotherapy.

Keywords: Thymidylate synthase (TS); dihydrofolate reductase (DHFR); mRNA; non-small cell lung cancer (NSCLC)

Submitted Sep 27, 2020. Accepted for publication Dec 01, 2020.

doi: $10.21037 /$ jtd-20-3185

View this article at: http://dx.doi.org/10.21037/jtd-20-3185 


\section{Introduction}

Lung cancer is the leading cause of cancer-related deaths worldwide, and is among the most common cancer types in both men and women (1). The reported number of annual deaths due to lung cancer is higher than that due to prostate, breast, and colon cancers combined (2). Nonsmall cell lung cancer (NSCLC) accounts for approximately $80 \%$ of all lung cancer cases, and more than $85 \%$ of patients are diagnosed at the advanced stage of this disease. The medium survival of patients with untreated advanced NSCLC is only 4-5 months (2). The overall 5-year survival for lung cancer in the USA is about $15 \%$, but it is lower in Europe, China, and developing countries (1). Platinumbased chemotherapy is the standard first-line treatment for patients with inoperable advanced NSCLC (3). However, a number of studies and meta-analyses have demonstrated that chemotherapy results in limited improvement in survival in patients with advanced NSCLC, and causes excessive toxicity.

Pemetrexed, a relatively new multi-target antifolate drug, inhibits the growth of various types of tumour, including advanced NSCLC (4). Previous trials have demonstrated that pemetrexed combined with cisplatin induces a superior response in patients with non-squamous cell carcinoma of NSCLC with a more significant decrease in the incidence of severe adverse events than cisplatin monotherapy $(3,5)$. Pemetrexed exerts its anti-tumour function by targeting three key folate-dependent enzymes, namely, thymidylate synthase (TS), dihydrofolate reductase (DHFR), and glycinamide ribonucleotide formyltransferase $(4,6)$. High levels of TS expression have been shown to correlate with reduced pemetrexed sensitivity in vitro (7). In addition, DHFR expression levels are reportedly associated with sensitivity to pemetrexed in patients with NSCLC (8).

Tumour tissues are the major source for biomarker examination, but given that they are heterogeneous and biopsy collection is inconvenient, levels of circulating nucleic acids have become a useful non-invasive tool for monitoring changes during cancer treatment (9). With the development of extremely sensitive detection methods, such as real-time PCR and high-throughput sequencing machines, cancer-related RNA molecules in plasma have become potential biomarkers for cancer diagnostics and prognostics $(10,11)$. Shen et al. (9) adopted a practical and convenient method for detecting plasma mRNA and demonstrated that the breast cancer susceptibility gene 1 (BRCA1) and TS mRNA levels in plasma mirror those in tumour tissues. In addition, they proposed that plasma BRCA1 and TS mRNA levels could be used as predictive biomarkers for docetaxel and pemetrexed responses in patients with gastric cancer (9). TS and DHFR expression levels in tumour tissues are associated with patient response to pemetrexed chemotherapy (12). Therefore, we investigated plasma TS and DHFR mRNA levels as potential biomarkers for identifying patients who may respond to and benefit the most from pemetrexed treatment. We present the following article in accordance with the REMARK reporting checklist (available at http:// dx.doi.org/10.21037/jtd-20-3185).

\section{Methods}

\section{Patients}

From July 2016 to December 2018, blood samples were collected from 245 patients diagnosed with NSCLC clinical stages III (including stages IIIA and IIIB) and IV, and 30 healthy controls (HCs) in Nantong Tumour Hospital (Nantong, China). All patients were treated with six cycles of pemetrexed/cisplatin at intervals of 21 days as the firstline chemotherapeutic strategy. The initial doses on the first day were $500 \mathrm{mg} / \mathrm{m}^{2}$ pemetrexed and $75 \mathrm{mg} / \mathrm{m}^{2}$ cisplatin. The patients were orally administered $4 \mathrm{mg}$ dexamethasone for 3 days, twice daily, starting from the day prior to chemotherapy, to reduce the incidence of severe adverse events. Seven days prior to chemotherapy, the patients were administered $1,000 \mathrm{~g}$ vitamin B12 and then once every 9 weeks until 21 days after the last pemetrexed dose. Moreover, the patients were orally administered $400 \mathrm{~g}$ folic acid/day for 7 days prior to chemotherapy until 21 days after the last pemetrexed dose.

The study was conducted in accordance with the Declaration of Helsinki (as revised in 2013). The study was approved by Medical Ethics Committee board of No. 2020A03 and informed consent was taken from all individual participants.

\section{Plasma mRNA expression detection}

Blood samples $(5 \mathrm{~mL})$ from each patient were collected in EDTA tubes. White blood cells were removed from whole blood by lymphocyte separation medium FicollPaque PLUS (GE Healthcare, USA). Total plasma RNA was extracted with Trizol following standard protocols. RNA integrity was evaluated by visualising the $18 \mathrm{~S}$ 
Table 1 Characteristics of NSCLC patients and healthy controls

\begin{tabular}{lcc}
\hline Characteristic & $\begin{array}{c}\text { NSCLC patients } \\
(\mathrm{n}=245)\end{array}$ & $\begin{array}{c}\text { Healthy controls } \\
(\mathrm{n}=30)\end{array}$ \\
\hline Age, years & $56.8 \pm 10.5$ & $58.2 \pm 11.6$ \\
Sex, $\mathrm{n}(\%)$ & $141(57.6)$ & $17(56.7)$ \\
Male & $104(42.4)$ & $13(43.3)$ \\
Female & & \\
Smoking status, $\mathrm{n}(\%)$ & $95(38.8)$ & $13(43.3)$ \\
Smoker & $150(61.2)$ & $17(56.7)$ \\
Non-smoker & & \\
Histology, $\mathrm{n}$ (\%) & $176(71.8)$ & \\
Adenocarcinoma & $46(18.8)$ & \\
Squamous cell carcinoma & $23(9.4)$ & \\
Large cell carcinoma & & \\
Disease stage (n, \%) & $47(19.2)$ & \\
IIIA & $71(29.0)$ & \\
IIIB & $127(51.8)$ & \\
IV & & \\
\hline
\end{tabular}

NSCLC, non-small cell lung cancer.

and $28 \mathrm{~S}$ RNAs via agarose gel electrophoresis. The RNA was transcribed into cDNA using the M-MLV reverse transcription kit (Promega, USA). Real-time PCR was performed to detect the expressions of TS and DHFR genes and the housekeeping gene $\beta$-actin. Gene expression was calculated relative to $\beta$-actin. Primer and probe sequences were as previously described (6). The primer sequences used were as follows: TS forward 5'-GAATCACATCGAGCCACTGAAA-3', reverse 5'-TTCGAAGA ATCCTGAGCTTTGG-3'; DHFR forward 5'-TAAACTGCATCGTCGCTGTGT-3', reverse 5 '-GGGCAGGTCCCCGTTCT-3'; $\beta$-actin forward 5'-AACTACCTTCAACTCCATCA-3', reverse 5'-GAGCAATGATCTTGATCTTCA-3'.

\section{Tumour response evaluation}

Baseline tumour assessments were performed within 28 days before chemotherapy. Tumour response to pemetrexed/ cisplatin chemotherapy was assessed according to the Response Evaluation Criteria in Solid Tumours (RECIST) v.1.0 as previously described $(3,4)$. The responses were divided into four categories: complete response (CR), partial response (PR), stable disease (SD), and progressive disease (PD). Overall survival (OS) was measured from the date of enrolment to the date of death as a result of any cause or date of data locking.

\section{Statistical analysis}

Data are presented as mean \pm standard deviation. Student's $t$-test was used to analyse differences between two groups. Pearson correlation coefficient was calculated to determine the correlation between TS and DHFR mRNA levels. Survival curve calculations were performed using the Kaplan-Meier method, and the difference in patient survival was determined by log-rank test. A P value $<0.05$ was considered statistically significant for all analyses.

\section{Results}

\section{Characteristics of patients}

The characteristics of the patients and HCs are shown in Table 1. Median age, sex, and smoking status were comparable between groups. Histologically, half of the patients $(51 \%)$ were diagnosed with adenocarcinoma, whereas $39.6 \%$ and $9.4 \%$ were diagnosed with squamous cell carcinoma and large cell carcinoma, respectively. Moreover, $38.2 \%$ of the patients were diagnosed with stage III NSCLC (IIIA: $19.2 \%$; IIIB: $29.0 \%$ ) and $51.8 \%$ were diagnosed with stage IV NSCLC.

\section{Plasma TS and DHFR mRNA levels in patients with advanced NSCLC}

The TS $(\mathrm{P}<0.001)$ and DHFR $(\mathrm{P}<0.01)$ mRNA levels were downregulated in patients with NSCLC compared with those in HCs (Figure 1A,B). Analysis of the relationship between plasma TS and DHFR mRNA levels in patients with advanced NSCLC revealed a significant correlation $(\mathrm{R} 2=0.4531, \mathrm{P}<0.0001)$ between their expression levels (Figure 1C).

\section{Response of plasma TS and DHFR mRNA levels to pemetrexed/cisplatin treatment}

The relationship between TS and DHFR mRNA levels and patient response to pemetrexed/cisplatin treatment was also determined. Treatment efficacy was evaluated for each patient in accordance with the RECIST criteria version 

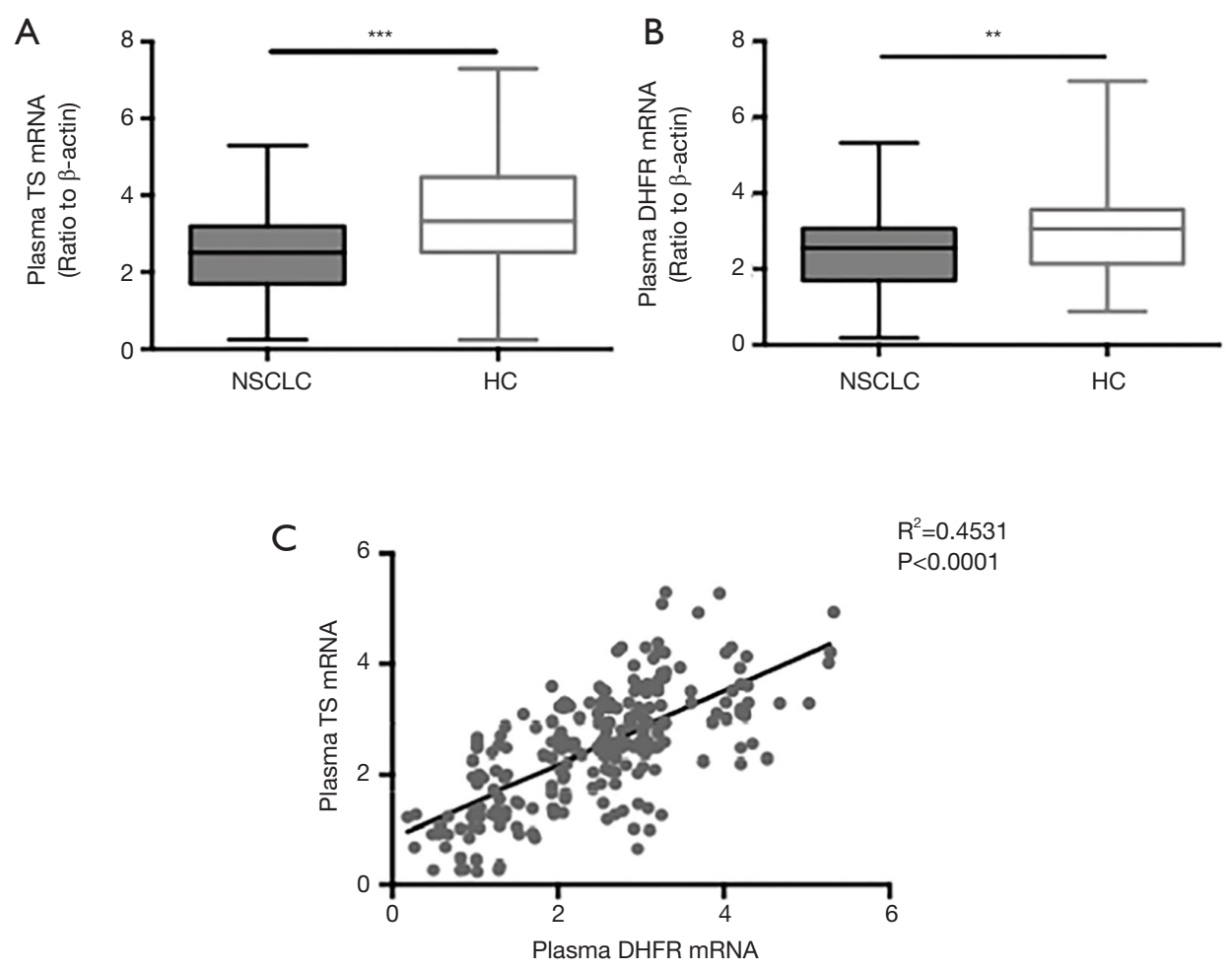

Figure 1 Plasma thymidylate synthase (TS) and dihydrofolate reductase (DHFR) mRNA levels. (A) TS and (B) DHFR mRNA expression levels in plasma samples of patients with non-small cell lung cancer (NSCLC) and heathy controls (HC). Relative mRNA levels were calculated relative to $\beta$-actin. Lines inside the boxes denote the medians. ${ }^{* *}, \mathrm{P}<0.01 ;{ }^{* *}, \mathrm{P}<0.001$. (C) Plasma TS and DHFR mRNA expression levels were significantly correlated in patients with NSCLC $\left(\mathrm{R}^{2}=0.4531, \mathrm{P}<0.0001\right)$.

Table 2 Summary of tumour response and plasma TS and DHFR mRNA levels

\begin{tabular}{lccc}
\hline Response & Case, $\mathrm{n}(\%)$ & TS mRNA & DHFR mRNA \\
\hline CR & $3(1.2)$ & $1.241 \pm 1.714$ & $1.105 \pm 0.887$ \\
PR & $96(39.1)$ & $2.081 \pm 0.846$ & $2.222 \pm 0.950$ \\
SD & $125(51.1)$ & $2.692 \pm 1.091$ & $2.544 \pm 1.096$ \\
PD & $21(8.6)$ & $2.972 \pm 0.747$ & $2.899 \pm 0.932$ \\
Response (CR + PR) & $99(40.4)$ & $2.056 \pm 0.862$ & $2.189 \pm 0.963$ \\
Non-response (SD + PD) & $146(59.6)$ & $2.732 \pm 1.051$ & $2.580 \pm 1.075$ \\
\hline
\end{tabular}

CR, complete response; DHFR, dihydrofolate reductase; PD, progressive disease; PR, partial response; SD, stable disease; TS, thymidylate synthase.

1.1. Overall pemetrexed/cisplatin response was defined as $\mathrm{CR}+\mathrm{PR}$, and 'non-response' was defined as PD + SD (Table 2). The mean TS mRNA level in the response and non-response groups was $2.056 \pm 0.862$ and $2.732 \pm 1.051$, respectively (Table 2, Figure $2 A$ ), and the respective mean DHFR mRNA were $2.189 \pm 0.963$ and $2.580 \pm 1.075$ (Table 2, Figure $2 B$ ). TS $(\mathrm{P}<0.0001)$ and $\mathrm{DHFR}(\mathrm{P}<0.01)$ mRNA levels significantly decreased in the response group compared with those in the non-response group (Figure 2A,B).

\section{Association between plasma TS and DHFR mRNA levels and OS following pemetrexed/cisplatin treatment}

The association between plasma TS and DHFR mRNA 

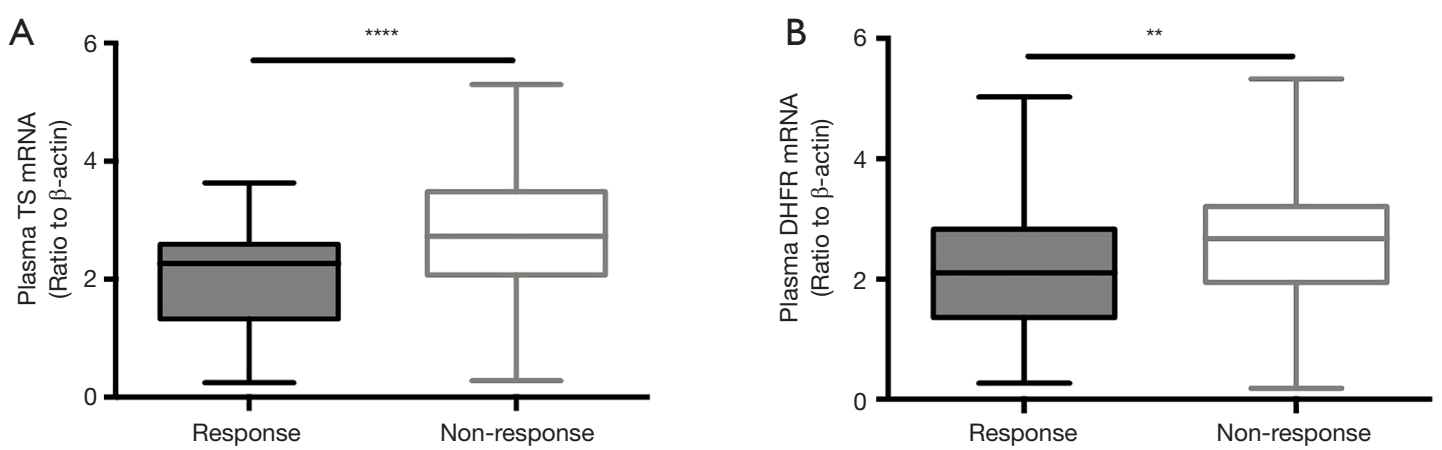

Figure 2 Association between plasma thymidylate synthase (TS) and dihydrofolate reductase (DHFR) mRNA levels and response to pemetrexed/cisplatin chemotherapy of patients with advanced NSCLC. Tumour response assessed using the Response Evaluation Criteria in Solid Tumours (RECIST) v.1.0. Response was defined as complete response (CR) + partial response (PR); non-response was defined as stable disease $(\mathrm{SD})+$ progressive disease $(\mathrm{PD})$. Lines inside the boxes denote the medians. ${ }^{* *}, \mathrm{P}<0.01 ;{ }^{* * *}, \mathrm{P}<0.0001$.
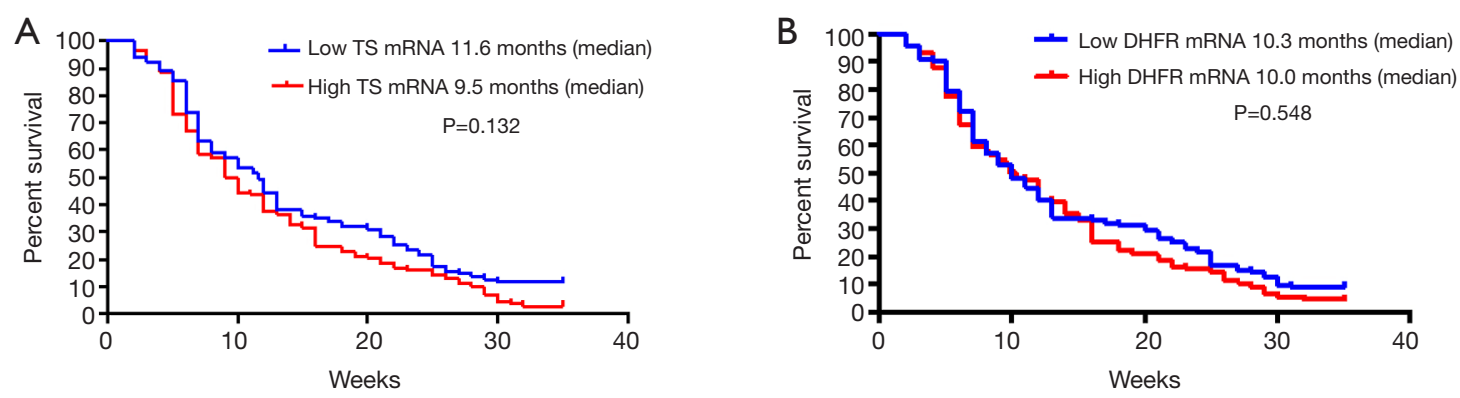

Figure 3 Kaplan-Meier survival curves of overall survival after pemetrexed/cisplatin chemotherapy. Median levels of (A) thymidylate synthase (TS) and (B) dihydrofolate reductase (DHFR) mRNA expressions used as the cut-off values for assigning patients into low/high mRNA groups.

levels and OS following pemetrexed/cisplatin treatment was determined. The mean relative TS and HDFR mRNA levels in the patient cohort were 2.46 and 2.42, respectively. The median levels of TS and HDFR mRNA expressions were used as the cut-off values for assigning low/high mRNA expression groups among the patients with cancer. Overall, patients with low TS mRNA levels had prolonged survival compared with those with high TS mRNA levels, although the difference was not statistically significant (11.6 vs. 9.5 months, respectively, $\mathrm{P}=0.132$; Figure $3 A$ ). Moreover, patients with low DHFR mRNA levels had similar survival times compared with those with high DHFR mRNA levels (10.3 vs. 10.0 months, respectively, $\mathrm{P}=0.548$; Figure $3 B$ ).

\section{Discussion}

In this study, plasma TS and DHFR mRNA levels decreased in patients with advanced NSCLC compared with those in HCs. Moreover, the plasma mRNA levels of these two genes were negatively correlated with tumour response to pemetrexed/cisplatin chemotherapy in patients with advanced NSCLC. Previous studies investigated the possible association between TS or DHFR expression levels and the response to pemetrexed monotherapy or combined pemetrexed and platinum chemotherapy in patients with NSCLC or other cancer types, including breast cancer $(4,6,13,14)$. In those studies, the TS and DHFR levels were determined in terms of tumour tissue mRNA levels $(13,14)$ or tumour tissue protein levels via immunohistochemistry and scoring $(4,5)$. A potential association between high TS mRNA levels in tumour tissues and ineffective responses to pemetrexed monotherapy has been suggested (13). In addition, tumour immunohistochemistry staining has revealed that high TS expression in tumour tissues inversely correlates with response to pemetrexed monotherapy in patients with NSCLC (4). The results of the present study 
suggested that TS gene expression is a promising biomarker for determining the clinical outcome of pemetrexed monotherapy or combined pemetrexed and platinum chemotherapy. Moreover, DHFR, the second target of pemetrexed, may also be a promising predictor of tumour response.

Although the expression levels of TS and DHFR in tumour tissues have been extensively studied, current knowledge on the relationship between the plasma mRNA levels of TS and DHFR genes and responses to pemetrexed monotherapy is limited. With the development of RNA enrichment and purification techniques and highly sensitive real-time PCR technology, the determination of gene expression has become feasible. Numerous studies have successfully determined the mRNA expressions of tumour-related genes, such as BRCA1 and TS (9), hTRRT $(10,15)$, beta-catenin (16), CXCR4 and Bmi-1 (17), in patients' plasma. Moreover, plasma TS and DHFR mRNA levels are highly associated with disease progression and prognosis in patients with cancer. In the present study, two pemetrexed targets, namely, TS and DHFR, in the form of plasma mRNA, were identified in patients with NSCLC. The results suggested that plasma TS and DHFR mRNAs have potential roles in predicting tumour response to pemetrexed/cisplatin therapy in patients with advanced NSCLC. In addition, the results confirmed that TS and DHFR mRNAs can be used to select patients who are likely to respond and benefit the most from chemotherapy.

Nevertheless, this study has several limitations. Although the correlation between plasma TS and DHFR mRNA levels and tumour response was established, the results did not indicate a significant difference in the OS of the patients, probably because of the small sample size. Moreover, the mRNAs were possibly packaged into exosomes and remained intact in the blood circulation, given that large amounts of RNAase exist in human blood. Thus, the total plasma mRNA in this study could not be conclusively confirmed as either free circulating mRNA or exosome-packaged mRNA. In future studies, circulating exosome mRNA must be ascertained if it is a more accurate biomarker than plasma total mRNA.

\section{Acknowledgments}

Funding: This study was supported by Methylation transferase METTL7B application and molecular mechanism research in early diagnosis of lung cancer; Research Fund for Young Medical Talents of Nantong City (WQ2016059) and Nanjing Science and Technology Bureau Project (YKK19176).

\section{Footnote}

Reporting Checklist: The authors have completed the REMARK reporting checklist. Available at http://dx.doi. org/10.21037/jtd-20-3185

Data Sharing Statement: Available at http://dx.doi. org/10.21037/jtd-20-3185

Conflicts of Interest: All authors have completed the ICMJE uniform disclosure form (available at http://dx.doi. org/10.21037/jtd-20-3185). The authors have no conflicts of interest to declare.

Ethical Statement: The authors are accountable for all aspects of the work in ensuring that questions related to the accuracy or integrity of any part of the work are appropriately investigated and resolved. The study was conducted in accordance with the Declaration of Helsinki (as revised in 2013). The study was approved by Medical Ethics Committee board of No. 2020-A03 and informed consent was taken from all individual participants.

Open Access Statement: This is an Open Access article distributed in accordance with the Creative Commons Attribution-NonCommercial-NoDerivs 4.0 International License (CC BY-NC-ND 4.0), which permits the noncommercial replication and distribution of the article with the strict proviso that no changes or edits are made and the original work is properly cited (including links to both the formal publication through the relevant DOI and the license). See: https://creativecommons.org/licenses/by-nc-nd/4.0/.

\section{References}

1. Dela Cruz CS, Tanoue LT, Matthay RA. Lung cancer: epidemiology, etiology, and prevention. Clin Chest Med 2011;32:605-44.

2. Schiller JH, Harrington D, Belani CP, et al. Comparison of four chemotherapy regimens for advanced non-smallcell lung cancer. N Engl J Med 2002;346:92-8.

3. Senan S, Brade A, Wang LH, et al. PROCLAIM: Randomized Phase III Trial of Pemetrexed-Cisplatin or 
Etoposide-Cisplatin Plus Thoracic Radiation Therapy Followed by Consolidation Chemotherapy in Locally Advanced Nonsquamous Non-Small-Cell Lung Cancer. J Clin Oncol 2016;34:953-62.

4. Komiya T, Memmott RM, Blumenthal GM, et al. A phase I/II study of pemetrexed with sirolimus in advanced, previously treated non-small cell lung cancer. Transl Lung Cancer Res 2019;8:247-57.

5. Uramoto H, Onitsuka T, Shimokawa H, et al. TS, DHFR and GARFT expression in non-squamous cell carcinoma of NSCLC and malignant pleural mesothelioma patients treated with pemetrexed. Anticancer Res 2010;30:4309-15.

6. Shimizu T, Nakanishi Y, Nakagawa Y, et al. Association between expression of thymidylate synthase, dihydrofolate reductase, and glycinamide ribonucleotide formyltransferase and efficacy of pemetrexed in advanced non-small cell lung cancer. Anticancer Res 2012;32:4589-96.

7. Giovannetti E, Backus HH, Wouters D, et al. Changes in the status of p53 affect drug sensitivity to thymidylate synthase (TS) inhibitors by altering TS levels. Br J Cancer 2007;96:769-75.

8. Jung M, Lee CH, Park HS, et al. Pharmacogenomic assessment of outcomes of pemetrexed-treated patients with adenocarcinoma of the lung. Yonsei Med J 2013;54:854-64.

9. Shen J, Wei J, Guan W, et al. Plasma mRNA expression levels of BRCA1 and TS as potential predictive biomarkers for chemotherapy in gastric cancer. J Transl Med 2014;12:355.

10. Terrin L, Rampazzo E, Pucciarelli S, et al. Relationship between tumor and plasma levels of hTERT mRNA in patients with colorectal cancer: implications for monitoring

Cite this article as: Li T, Huang JB, Lu JG, Li R, Wang Y, Shi XR, Shi MX, Zhang XD. Plasma thymidylate synthase and dihydrofolate reductase mRNA levels as potential predictive biomarkers of pemetrexed sensitivity in patients with advanced non-small cell lung cancer. J Thorac Dis 2020;12(12):7313-7319. doi: $10.21037 /$ jtd-20-3185 of neoplastic disease. Clin Cancer Res 2008;14:7444-51.

11. Fleischhacker M, Schmidt B. Free circulating nucleic acids in plasma and serum (CNAPS) -- Useful for the detection of lung cancer patients? Cancer Biomark 2010;6:211-9.

12. Shimizu T, Nakanishi Y, Nakagawa Y, et al. Association between expression of thymidylate synthase, dihydrofolate reductase, and glycinamide ribonucleotide formyltransferase and efficacy of pemetrexed in advanced non-small cell lung cancer. Anticancer Res 2012;32:4589-96.

13. Gomez HL, Santillana SL, Vallejos CS, et al. A phase II trial of pemetrexed in advanced breast cancer: clinical response and association with molecular target expression. Clin Cancer Res 2006;12:832-8.

14. Righi L, Papotti MG, Ceppi P, et al. Thymidylate synthase but not excision repair cross-complementation group 1 tumor expression predicts outcome in patients with malignant pleural mesothelioma treated with pemetrexedbased chemotherapy. J Clin Oncol 2010;28:1534-9.

15. Perhavec A, Cerkovnik P, Novakovic S, et al. The hTERT mRNA in plasma samples of early breast cancer patients, non-cancer patients and healthy individuals. Neoplasma 2008;55:549-54.

16. Wong SC, Lo SF, Cheung MT, et al. Quantification of plasma beta-catenin mRNA in colorectal cancer and adenoma patients. Clin Cancer Res 2004;10:1613-7.

17. $\mathrm{Xu}$ W, Zhou H, Qian H, et al. Combination of circulating CXCR4 and Bmi-1 mRNA in plasma: A potential novel tumor marker for gastric cancer. Mol Med Rep 2009;2:765-71.

(English Language Editor: J. Jones) 